\title{
Efficiency of oxidative work performance of skeletal muscle in patients with cystic fibrosis
}

\author{
K de Meer, J A L Jeneson,* V A M Gulmans, J van der Laag, $\mathrm{R}$ Berger
}

\begin{abstract}
Background - Exercise intolerance in patients with cystic fibrosis is commonly attributed to reduced pulmonary and nutritional status. The possible role of diminished efficiency of mitochondrial oxidative phosphorylation in relation to skeletal muscle performance was investigated in patients with cystic fibrosis. Methods - In vivo synthesis of ATP in skeletal muscle during submaximal exercise was studied in eight patients with cystic fibrosis aged 12-17 years, and in 19 healthy control subjects aged 8-36 years. The intracellular $\mathrm{pH}$ and concentrations of phosphate compounds were calculated at four steady states from phosphorus-31 labelled nuclear magnetic resonance spectroscopy measurements in the forearm muscle during bulb squeezing in an exercise protocol. Normalised power output, expressed as percentage maximal voluntary contraction ( $Y$, in \%MVC), was related to the energy force of ATP hydrolysis (X $=\ln [$ [ATP]/[ADP][Pi]). This relationship provides an in vivo measure of efficiency of oxidative work performance of skeletal muscle.
\end{abstract}

Results - During all workloads (but not at rest) intracellular $\mathrm{pH}$ was higher in the patients with cystic fibrosis than in the controls. The linear least square fit for $Y=$ a - bX showed high correlations in both groups; the slope b was $19 \%$ lower in the patients than in the controls $(11.8 \% v 14.5 \%$ MVC/ln M; $95 \%$ confidence interval for difference $0 \cdot 3$ to $5 \cdot 0$ ).

Conclusions - In patients with cystic fibrosis oxidative work performance of skeletal muscle is reduced. This may be related to secondary pathophysiological changes in skeletal muscle in cystic fibrosis.

(Thorax 1995;50:980-983)

Keywords: cystic fibrosis, exercise, mitochondria, oxidative phosphorylation.

Respiratory infections and chronic airways obstruction are frequent problems in patients with cystic fibrosis and may cause lung damage and respiratory insufficiency. When the clinical condition declines, exercise intolerance is frequently seen. This is associated with severity of pulmonary disease (decrease of forced expiratory volume in one second) and with the nutritional status in these patients. ${ }^{12}$ In patients with chronic obstructive pulmonary disease similar associations between weight loss and chronic obstructive bronchitis have also been related to exercise intolerance. ${ }^{34}$ However, the pathophysiology of decreased exercise intolerance in patients with chronic obstructive pulmonary disease and in patients with other causes of chronic airways obstruction is still not completely understood. Recent studies indicate that intrinsic abnormalities of skeletal muscle may contribute to the limited exercise endurance in these patients. Investigations of in vivo oxidative capacity in calf ${ }^{5}$ and forearm ${ }^{6}$ muscle in adults with chronic obstructive pulmonary disease have shown decreased capacity, reflected by a significant intracellular acidosis and depletion of phosphocreatine (PCr). It has been postulated that such changes may be due to differences in oxygen supply during exercise, as well as to muscle composition, nutritional status and activity levels, or a combination of these, rather than intrinsic defects in the synthesis of mitochondrial adenosine triphosphate (ATP). ${ }^{56}$

However, in patients with cystic fibrosis studies of fibroblasts and leucocytes have shown mitochondrial abnormalities including an increased calcium concentration, ${ }^{7}$ lower $\mathrm{NADH}$ dehydrogenase (respiratory chain enzyme complex I) activity, ${ }^{8}$ and higher $\mathrm{pH}$ optimum of $\mathrm{NADH}$ dehydrogenase ${ }^{9}$ compared with controls. At present the impact of the reduced pulmonary and nutritional status in patients with clinically advanced cystic fibrosis and the in vitro abnormalities of mitochondria from cell cultures of patients with cystic fibrosis on the in vivo oxidative capacity of the muscles of these patients and on the bioenergetics of their muscle cells during exercise remains unclear. We therefore investigated the in vivo mitochondrial performance during exercise of peripheral skeletal muscle in children and adolescents with cystic fibrosis.

\section{Methods}

SUBJECTS

The patient group consisted of eight patients (three male) of mean (SD) age 14 (2) years (range 12-17) with cystic fibrosis, whose diagnosis was confirmed by a positive sweat test. Treatment consisted of pancrelipase substitution, nutritional surveillance, vitamin supplements (A, D, E, and K), physiotherapy, and antibiotics when indicated. None of the patients received supplemental oxygen. All patients attended school and all but one were engaged in recreative and school sport activities. All had some evidence of their disease (as shown by their Shwachman score ${ }^{10}$ ), signs of airways obstruction (forced expiratory volume in one second or forced vital capacity $<80 \%$ predicted) 
Table 1 Clinical features of subjects with cystic fibrosis $(n=8)$

\begin{tabular}{|c|c|c|c|}
\hline & Mean & (SD) & Range \\
\hline $\begin{array}{l}\text { Shwachman score (normal }=100) \\
\text { Weight for age }\left(\% \text { of median }{ }^{23}\right) \\
\text { Height for age }\left(\% \text { of } \text { median }^{23}\right) \\
\text { FEV }_{1}\left(\% \text { predicted }{ }^{24}\right) \\
\text { FVC }\left(\% \text { predicted }{ }^{24}\right) \\
\text { Transcutaneous oxygen saturation }(\%)\end{array}$ & $\begin{array}{l}64 \\
7 \cdot 6 \\
31 \\
61 \\
74 \\
96\end{array}$ & $\begin{array}{l}(14) \\
(4 \cdot 1) \\
(31) \\
(19) \\
(17) \\
(1)\end{array}$ & $\begin{array}{r}45-85 \\
3-16 \\
2-93 \\
29-85 \\
50-98 \\
94-98\end{array}$ \\
\hline $\begin{array}{l}\text { Capillary blood gas analysis: } \\
\text { pH } \\
\mathrm{PCO}_{2}(\mathrm{kPa}) \\
\text { Base excess (mM) }\end{array}$ & $\begin{array}{l}7 \cdot 38 \\
6 \cdot 1 \\
-0.6\end{array}$ & $\begin{array}{l}(0.03) \\
(0.5) \\
(1 \cdot 2)\end{array}$ & $\begin{array}{l}7 \cdot 36-7 \cdot 43 \\
5 \cdot 4-7 \cdot 1 \\
-2 \cdot 3-+0 \cdot 9\end{array}$ \\
\hline
\end{tabular}

$\mathrm{FEV}_{1}=$ forced expiratory volume in one second; $\mathrm{FVC}=$ forced vital capacity.

and/or a diminished nutritional status (height for age under the third centile, or weight for height under the tenth centile). The transcutaneous oxygen saturation (measured with a Nellcor pulse oximeter) was $<95 \%$ in two patients. Patient data are summarised in table 1. The control group comprised 19 subjects (14 male) of mean (SD) age 22 (7) years (range 8-36) with no history of recurrent respiratory disease or dyspnoea, and with a sedentary lifestyle. This group included six children younger than 18 years (mean age 12 years), and 13 adults (mean age 26 years).

The study was approved by the medical ethical committee of our hospital and informed consent was obtained from all subjects.

\section{THEORETICAL MODELLING}

For a quantitative analysis of phosphorus-31 nuclear magnetic resonance $\left({ }^{31} \mathrm{P}-\mathrm{NMR}\right)$ measurements in human skeletal muscle a thermodynamic model of adenine nucleotide feedback control of oxidative phosphorylation was used as previously described. ${ }^{11}$ Briefly, the model is based on the theory of mosaic non-equilibrium thermodynamics ${ }^{12}$ which proposes that, with certain assumptions such as negligible contribution of glycolytic ATP production, the mitochondrial-driven ATP flow can be described by a quasilinear function of the free energy force. This energy force of cytosolic ATP hydrolysis can be derived from the substrate and product concentrations in mitochondrial ATP production as mathematically expressed by the logarithm of the phosphorylation potential $^{1314}$ - that is, ln [ATP]/
$[\mathrm{ADP}][\mathrm{Pi}]$ - where $[\mathrm{Pi}]$ is the concentration of inorganic phosphate and [ATP] and [ADP] are the concentrations of ATP and adenosine diphosphate (ADP). Under steady state conditions and constant $\mathrm{pH} 7 \cdot 0$ this "energy forceflow" relation can be accurately approximated by a linear relationship for $80 \%$ of the range of ATP production. ${ }^{112}$ Assuming that muscle ATP consumption increases proportionally with power output, ${ }^{15}$ relations between different workloads and thermodynamically controlled oxidative phosphorylation in mitochondria in recruited muscle fibres during in vivo ${ }^{31} \mathrm{P}-\mathrm{NMR}$ spectroscopy can thus be described as:

Power output $=a-b \times \ln [\mathrm{ATP}] /[\mathrm{ADP}] \times[\mathrm{Pi}]$

where $a$ and $b$ are constants. Hence, differences in the slope $b$ and in power output between patients and control subjects at given values of energy force are an in vivo measure of mitochondrial efficiency in terms of work performance by the sampled muscle fibres.

\section{NMR EXPERIMENTS}

The study was conducted on the flexor digitorum profundus muscle of the right and dominant forearm in all subjects using a 1.5 Tesla NMR spectrometer (Philips S15 HP) at a frequency of $25.86 \mathrm{MHz}$, as previously described. ${ }^{16}$ Briefly, measurements of four minutes were conducted at four steady states: at rest and during exercise (involving bulb squeezing using only the fourth and fifth finger with a repetition time of three seconds) at three normalised workloads. Power output was measured and recorded as $\mathrm{dPdV}$ (developed pressure times displaced volume of air) and normalised to power output during maximal voluntary contraction (MVC) of the forearm muscle. Workloads were normally aimed at $20 \%, 40 \%$, and $50 \%$ MVC. Data were analysed as previously described. ${ }^{16}$ The concentrations of inorganic phosphate and of phosphocreatine ([Pi] and $[\mathrm{PCr}]$ ) were calculated from the measured relative changes in $\mathrm{Pi}$ and $\mathrm{PCr}$ from resting levels, and the free [ADP] was calculated from the creatine kinase equi-
A

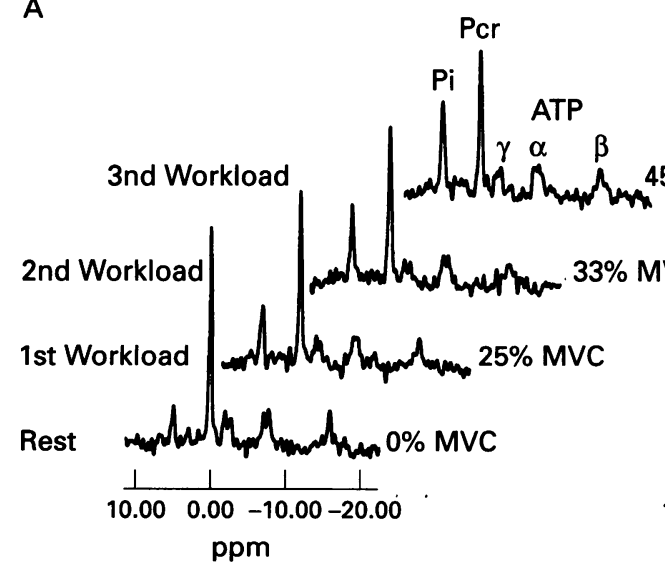

B

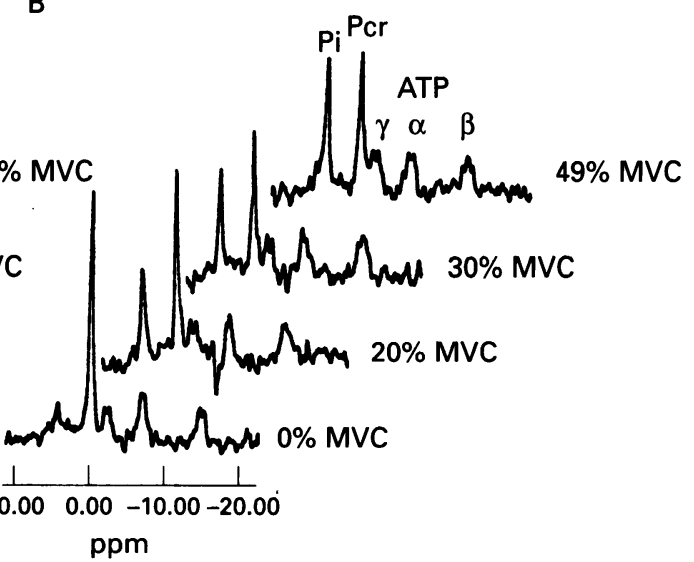

Figure 1 Two series of ${ }^{31}$ P-NMR spectra of flexor digitorum profundus muscle obtained from (A) an adult control subject and $(B)$ a patient with cystic fibrosis at rest and during exercise. Note the changes of inorganic phosphate (Pi) relative to phosphocreatine (PCr) in comparison with measured power output (\%MVC) at different workloads. 


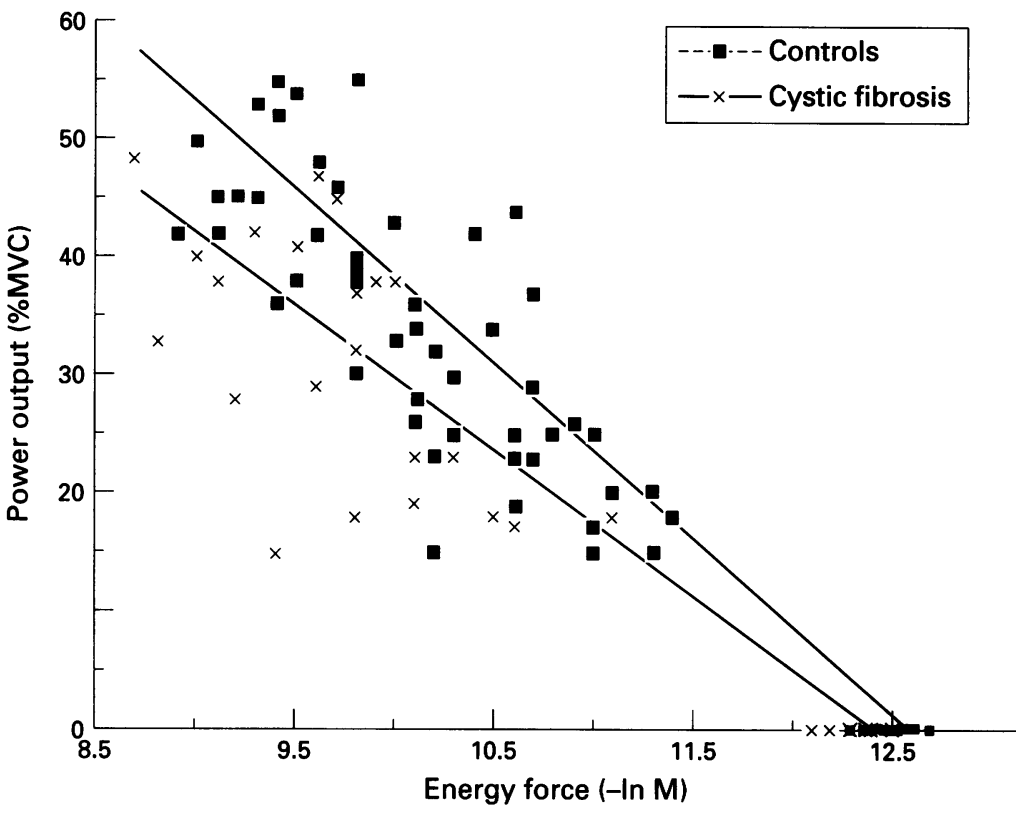

Figure 2 Free energy force and power output of flexor digitorum profundus muscle fibres at rest and during exercise in patients with cystic fibrosis (31 steady states in eight subjects) and controls (71 steady states in 19 subjects) with the regression lines of the linear least squares fit in both groups.

librium relation - that is, $[\mathrm{ADP}]=([\mathrm{ATP}] \times$ [creatine] $) /\left(1.66 \times 10^{9} \times 10^{-\mathrm{pH}} \times[\mathrm{PCr}]\right)$, where [creatine] is the free creatine concentration. ${ }^{17}$ Intracellular $\mathrm{pH}\left(\mathrm{pH}_{\mathrm{i}}\right)$ was calculated from the chemical shift between the $\mathrm{Pi}$ and $\mathrm{PCr}$ peaks. ${ }^{18}$ Data of eight measurements were discarded because intracellular $\mathrm{pH}_{\mathrm{i}}$ was $<6.90$, indicating that the condition of constant $\mathrm{pH} 7 \cdot 0$ was no longer met.

\section{STATISTICAL ANALYSIS}

Comparisons of $\mathrm{pH}_{\mathrm{i}}$ between the groups were made with Student's $t$ test. Muscular work was calculated by fitting linear least square models of the energy force and power output to the combined series of measurements within each group. Comparisons were made by calculating confidence intervals (CI) for between group differences in slopes and vertical distance between the regression lines, as described by Altman and Gardner. ${ }^{19}$ Statistical differences were considered significant if $\mathrm{p}<0.05$ (twotailed).

Table 2 Least squares fit for the thermodynamic modelt of free energy force and power output in flexor digitorum profundus muscle of patients with cystic fibrosis and control subjects studied with ${ }^{31} P-N M R$ spectroscopy in the exercise protocol

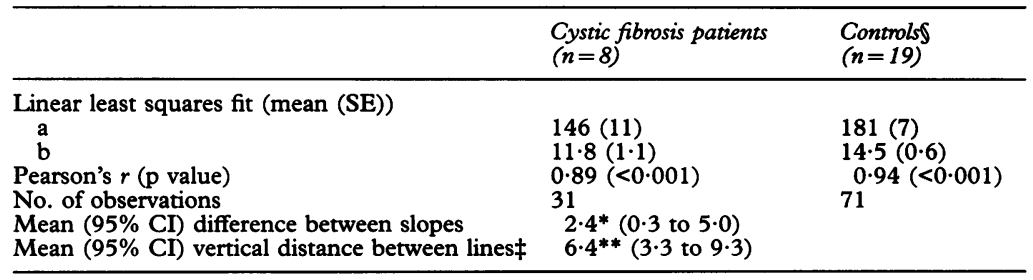

$* \mathrm{p}<0.05 ;{ }^{* *} \mathrm{p}<0.001$ ( $t$ test)

†The linear model of power output (expressed as \%MVC, see text) and free energy force (expressed as - In M, that is the natural logarithm of the concentrations in mol/l (M) of the relevant phosphate metabolites, i.e. $\mathrm{Y}=\mathrm{a}-\mathrm{b} \times \ln [\mathrm{ATP}] /[\mathrm{ADP}][\mathrm{Pi}]$ ), was fitted to the pooled

measurements in each group.

Vertical distance between the regression lines of patients and control subjects at the mean of the energy force in the control group (i.e. $X=10.74-\ln M$, corresponding with a fitted $Y$ in the control group of $25 \%$ MVC)

For controls of $<18$ years $\mathrm{Y}=164-13.1 \times \mathrm{X} \quad(r=0.95)$; for controls of $>18$ years $\mathrm{Y}=$ $184-14.7 \times \mathrm{X}(r=0.94)$; no statistical difference in slope and vertical distance.

\section{Results}

Figure 1 depicts the NMR spectra of a patient with cystic fibrosis and a control subject. Changes in $\mathrm{Pi}$ and $\mathrm{PCr}$ levels in the muscle at comparable power output levels are clearly more pronounced in the patient than in the control subject. This indicates that a greater perturbation of the cellular energy force is required in the patient to generate a similar amount of power.

The results of calculated free energy force and power output of the sampled forearm muscle for all measurements in both groups of subjects and the linear regression lines are depicted in fig 2 . Linear least squares fit comparisons of muscle performance in the muscle are given in table 2. Pearson correlation coefficients indicated that a good fit was obtained for both groups $(p<0.001)$. The slope of the linear regression equation in this model for the energy force-flow relationship of patients with cystic fibrosis was $19 \%$ lower than in the controls $(b=11 \cdot 8 \%$ versus $14.5 \% \quad M V C / l n \quad M$ respectively, $\mathrm{p}<0 \cdot 05)$. The vertical distance between the linear regression lines at the mean value of free energy force in the control subjects $(10.74-\ln M)$ is another parameter reflecting efficiency of oxidative work performance in the sampled muscle. At the fitted power output level of approximately $25 \% \mathrm{MVC}$ in the control subjects the patients with cystic fibrosis sustained an output of $19 \%$ MVC, demonstrating a relative decrease of $25 \%$ in power output in the patients compared with the controls $(p<0.001)$. Likewise, the vertical distance at the mean value of the free energy force was also significantly lower in the patients than in the six age-matched control subjects $(p<0.05)$. Within the control group no significant differences in mitochondrial performance were found between the subjects aged more than or less than 18 years.

The calculated intracellular $\mathrm{pH}$ at rest did not differ between patients with cystic fibrosis and controls, but during exercise $\mathrm{pH}_{\mathrm{i}}$ was higher in the patients than in the controls $(\mathrm{p}<0.01)$. Acidosis $\left(\mathrm{pH}_{\mathrm{i}}<6.90\right)$ did not occur in any of the patients during exercise.

\section{Discussion}

In vivo ${ }^{31} \mathrm{P}-\mathrm{NMR}$ spectroscopy during exercise can be used to study the efficiency of mitochondrial ATP synthesis from oxidative phosphorylation in relation to work performance of skeletal muscle. In the present study we applied a quantitative method ${ }^{11}$ to measure mitochondrial performance which corrects for differences in nutritional status between subjects. For that purpose, workloads in the exercise protocol were tailored to individual maximal power output of the sampled muscle. In previous ${ }^{31} \mathrm{P}-\mathrm{NMR}$ spectroscopy studies of skeletal muscle metabolism in patients with chronic obstructive pulmonary disease ${ }^{5620}$ other parameters of mitochondrial capacity were studied and they were not always tailored to individual maximal power output.

In our protocol the metabolic status of the forearm muscle sampled with ${ }^{31} \mathrm{P}-\mathrm{NMR}$ spec- 
troscopy was related to sustained power output using a thermodynamic model of ATP production. This approach showed excellent linear least square fits in the patients with cystic fibrosis as well as in the control subjects, and is in agreement with other experiments and theoretical expectations. ${ }^{11221}$ Statistically significant differences in slope and power output at comparable and arbitrary levels of free energy were found, indicating that the efficiency of oxidative ATP synthesis in exercising forearm muscle was $19-25 \%$ lower in the patients with cystic fibrosis than in healthy control subjects.

Before we draw conclusions on the clinical relevance of these findings, we should address whether the results could be due to experimental bias. In our thermodynamic model we assumed that changes in the redox state of the muscle cells do not substantially contribute to control of the rate of mitochondrial ATP synthesis. ${ }^{21}$ Under hypoxic conditions and during exercise the concentrations of pyruvate and $\mathrm{NADH}$ in the cell could change the cytosolic redox state and activation of the lactate dehydrogenase complex with increased lactate production would ensue. With regard to the former, hypoxaemia was not a concern in the patients with cystic fibrosis. Although the resting oxygen saturation in the patients did not reach $100 \%$ (table 1 ), the actual levels of resting oxygen saturation exceeded the Michaelis constant of mitochondria for oxygen more than tenfold. ${ }^{22}$ Also, blood gas tensions (and plasma lactate concentrations, unpublished data) at rest in all patients were within normal limits. With regard to the latter, the absence of a severe drop in $\mathrm{pH}_{\mathrm{i}}$ during exercise indicates that no substantial lactate production occurred over the range of workloads studied (maximal $\mathrm{pH}_{\mathrm{i}}$ change $=0 \cdot 1$ units in patients and controls).

The main finding of our study was that the efficiency of skeletal muscle to perform work was up to $25 \%$ lower in the patients with cystic fibrosis than in controls (figs 1 and 2). Since our measurements addressed the balance between ATP free energy utilisation by the contractile elements and ATP free energy production by the mitochondria, the result may be explained by a diminished efficiency of the process on either side of the free energy balance. With respect to a diminished efficiency of force generation during contraction, we cannot exclude this explanation of our findings. However, with regard to impaired in vivo mitochondrial function in skeletal muscle of patients with cystic fibrosis, evidence for mitochondrial abnormalities has been reported in the literature. Specifically, in an in vitro study of leucocyte mitochondria in patients with cystic fibrosis Dechecci and coworkers found that properties of complex I of the respiratory chain were significantly altered. ${ }^{8}$ Changes in mitochondrial function in patients with cystic fibrosis may therefore be secondary to their diminished clinical status - for example, activity levels - or nutritional status - for example, rate of protein synthesis.

The gene responsible for the clinical manifestations in cystic fibrosis (the cystic fibrosis transmembrane conductance regulator involved in transmembrane chloride transport in epithelial tissues) has not until now been shown to be expressed in skeletal muscle. It seems unlikely that the reduced efficiency of work performance in the forearm muscle of patients with cystic fibrosis could be attributed to a primary effect of the mutated gene.

In conclusion, the results of this study indicate that efficiency of oxidative work performance of skeletal muscle in patients with cystic fibrosis is reduced by $19-25 \%$. A decrease in mitochondrial function secondary to clinical or nutritional factors which affects mitochondrial density and metabolism may be the explanation for this finding. Further studies of muscle mitochondrial function in patients with cystic fibrosis at different stages of this disease and at higher levels of work output are indicated.

The authors thank the Department of Radiology of the University Hospital in Utrecht for the use of the NMR facilities.

1 Neijens HJ, Duiverman EJ, Kerrebijn KF, Sinaasappel M Influence of respiratory exacerbation on lung function variables and nutritional status in CF patients. Acta Paediatr Scand 1985;317:38-41.

2 Marcotte JE, Canny GJ, Grisdale R, Desmond K, Corey $M$, Zinman $R$, et al. Effects of nutritional status on exercise performance in advanced cystic fibrosis. Chest 1986;90: 375-9.

3 Young IH, Woolcock AJ. Arterial blood gas tension changes at the start of exercise in chronic obstructive pulmonary disease. Am Rev Respir Dis 1979;119:213-21.

4 Loke JK, Mahler DA, Paul Man SF, Wiedemann HP, Matthay RA. Exercise impairment in chronic obstructive pulmonary disease. Clin Chest Med 1984;5:121-43.

5 Thompson CH, Davies RJO, Kemp GJ, Taylor DJ, Radda GK, Rajagopalan B. Skeletal muscle metabolism during exercise and recovery in patients with respiratory failure. Thorax 1993;48:486-90.

6 Kutsuzawa T, Shioya S, Kurita D, Haida M, Ohta Y, Yamabayashi H. ${ }^{31}$ P-NMR study of skeletal muscle metabolism in patients with chronic respiratory impairment. Am Rev Respir Dis 1992;146:1019-24.

7 Shapiro BL. Evidence for a mitochondrial lesion in cystic fibrosis. Life Sci 1989;44:1327-34.

8 Dechecchi MC, Girella E, Cabrini G, Berton G. The Km of NADH dehydrogenase is decreased in mitochondria of cystic fibrosis cells. Enzyme 1988;40:45-50.

9 Shapiro BL, Feigal RJ, Lam LFH. Mitochondrial NADH dehydrogenase in cystic fibrosis. Proc Natl Acad Sci USA 1979;76:2979-83.

10 Shwachman H, Kulczycki LL. Long-term study of one hundred five patients with cystic fibrosis. Am $\mathcal{F}$ Dis Child 1958;96:6-15.

11 Jeneson JAL, Westerhoff HV, Brown TR, van Echteld CJA, Berger R. Quasi-linear relationship between Gibbs free energy of ATP hydrolysis and power output in human forearm muscle. Am f Physiol 1995;268:C1474-84.

12 Westerhof HV, van Dam K. Thermodynamics and control of free-energy transduction. Amsterdam: Elsevier, 1987.

13 Lehninger AL. Biochemistry. 2nd edn. New York: Worth Publishers, 1976:539-40.

14 Rosing J, Slater EC. The value of delta $\mathrm{G}^{\circ}$ for the hydrolysis of ATP. Biochim Biophys Acta 1972;267:275-90.

15 Pybus J, Tregear RT. The relationship between adenosine triphosphatase activity to tension and power output of insect flight muscle. $\mathcal{F}$ Physiol 1975;247:71-89.

16 Jeneson JAL, van Dobbenburgh JO, van Echteld CJA, Lekkerkerk C, Janssen WJM, Dorland L, et al. Experimental design of ${ }^{31} \mathrm{P}$ MRS assessment of human forearm muscle design of ${ }^{31} \mathbf{P}$ MRS assessment of human forearm muscle function: restrictions imposed by

17 Meyer RA, Kushmerick MJ, Brown TR. Application of ${ }^{31}$ P-NMR spectroscopy to the study of striated muscle metabolism. Am ₹ Physiol 1982;242: C1-11.

18 Taylor DJ, Bore PJ, Styles P, Gadian DG, Radda GK Bioenergetics of intact human muscle. A ${ }^{31} \mathrm{P}$ nuclear magnetic resonance study. Mol Biol Med 1983;1:77-94.

19 Altman DG, Gardner MJ. Calculating confidence intervals for regression and correlation. BMf 1988;296:1238-42.

20 Payen RL, Wuyam B, Reutenauer H, Laurent D, Levy P, Le Bas J-F, et al. Impairment of muscular metabolism in Le Bas J-F, et al. Impairment of muscular metabolism in
chronic respiratory failure. A human ${ }^{31} \mathrm{P}$ MRS study. NMR
Biomed 1991:4:41-5.

21 Westerhof HV, van Echteld CJA, Jeneson JAL. On the expected relationship between Gibbs free energy of ATP hydrolysis and muscle performance. Biophys Chem 1995; 54:137-42.

22 Balaban RS. Regulation of oxidative phosphorylation in the mammalian cell. Am $\mathcal{F}$ Physiol 1990;258:C377-89.

23 Anonymous. Growth curves for children birth-18 years. Washington, DC: National Center for Health Statistics, 1977.

24 Zapletal A, Samanek M, Paul T. Lung function in children and adolescents; methods and reference values. Progress in respiratory disease. Basel: Herzog, 1987. 